\title{
Efficiency of a Multilayer-Laue-Lens with a 102 micron Aperture
}

\author{
Albert T. Macrander ${ }^{1, a}$, Adam Kubec ${ }^{1,3}$, Raymond Conley ${ }^{1,2}$, \\ Nathalie Bouet ${ }^{2}$, Juan Zhou ${ }^{2}$, Michael Wojcik ${ }^{1}$, and Jorg Maser ${ }^{1}$ \\ ${ }^{1}$ Advanced Photon Source, Argonne National Laboratory, Argonne, Illinois, 60439, USA \\ ${ }^{2}$ NSLS-II, Brookhaven National Laboratory, Upton, New York 11973, USA and \\ ${ }^{3}$ Fraunhofer IWS Dresden, Winterbergstr. 28, 01277 Dresden, Germany
}

(Dated: August 3, 2015)

\begin{abstract}
A multilayer-Laue-lens (MLL) comprised of $\mathrm{WSi}_{2} / \mathrm{Al}$ layers stacked to a full thickness of 102 microns was characterized for its diffraction efficiency and dynamical diffraction properties by x-ray measurements made in the far field. The achieved aperture roughly doubles the previous maximum reported aperture for an MLL, thereby doubling the working distance. Negative and positive first orders were found to have $14.2 \%$ and $13.0 \%$ efficiencies, respectively. A section thickness of 9.6 $\mu \mathrm{m}$ was determined from Laue-case thickness fringes in the diffraction data. A background gas consisting of $90 \% \mathrm{Ar}$ and $10 \% \mathrm{~N}_{2}$ was used for sputtering. This material system was chosen to reduce grown-in stress as the multilayer is deposited. Although some regions of the full MLL exhibited defects, the presently reported results were obtained for a region devoid of defects. The data compare well to dynamical diffraction calculations with Coupled Wave Theory (CWT) which provided confirmation of the optical constants and densities assumed for the CWT calculations.
\end{abstract}

Many experiments at synchrotrons require nanofocusing at high x-ray energies with a high flux of photons in the focus. One desires not only a lens that focuses efficiently, but also one that provides a reasonably large working distance. MLLs can achieve nanofocusing because the outer most layer thickness of the linear zone plate structure, which determines the spatial resolution, is limited only by the technology of thin film sputtering [1-8], and a sputtered layer thickness less than $0.7 \mathrm{~nm}$ has been demonstrated [9]. Furthermore, focusing efficiencies can be dramatically increased by satisfying a Bragg condition [10]. However, apertures of MLLs are limited by the achievable multilayer stack thickness. In this regard, overcoming the consequences of built-up stress is a challenge. The present work demonstrates an aperture of roughly twice the largest previously reported aperture for an MLL. This was accomplished with a bilayer material system not previously used for an MLL. Largest apertures reported to-date are $43 \mu \mathrm{m}$ [11] and $53 \mu \mathrm{m}$ [12].

The MLL was comprised of 15170 individual layers with Fresnel zones numbered 15802 down to 632 and paired as bilayers. Two layers in each bilayer were of equal thickness. The interfaces were parallel by design. The first-to-grow layer was $\mathrm{WSi}_{2}$ grown on a $\mathrm{Si}$ substrate with a deposited thickness of $4 \mathrm{~nm}$. This layer formed the outermost zone. Subsequent zones with increasing thickness were deposited until $80 \%$ of a single-sided zone plate structure was fabricated. After deposition a sectioned portion was cut-out and thinned to form a lens useable for experiments [3]. The lens was $2.7 \mathrm{~mm}$ long (in the out-of-the-page direction in Fig. 1) with portions along this length that were deformed by bending and also exhibited abrupt changes in angle between adjacent zones at some locations [13]. The present study is for a section of $0.17 \mathrm{~mm}$ located at the extreme end of the $2.7 \mathrm{~mm}$ length which was found to be devoid of such defects.

Diffraction measurements as a function of rocking angle were made at beamline 1-BM at the Advanced Photon Source [14]. The MLL was mounted in a prototype microscope [15]. The photon energy was set to $12 \mathrm{keV}$ by the main beamline monochromator with a $\mathrm{Si}(111)$ double crystal arrangement. The full lens aperture was set to diffract onto a Pilatus $100 \mathrm{~K}$ area detector placed $900 \mathrm{~mm}$ downstream of the lens. The experimental arrangement is shown in Fig.1. The Pilatus detector was operated at low gain and $50 \%$ threshold. The incident flux was reduced so that the direct beam count rate was $75 \mathrm{kHz}$, and counting times were 5 sec. Under these conditions a dead-time correction to the measured count rate is negligible [16]. Both negative (focusing) and positive (defocusing) first order diffraction patterns were recorded. The pixel size of this detector was $172 \mu \mathrm{m}$, with negligible space between pixels [16]. The negative and positive first orders were recorded in far-field patterns after a real downstream focus and a virtual upstream focus, respectively. However, because the focal length of $9.6 \mathrm{~mm}$ at 12 $\mathrm{keV}$ was far less than far-field location of the detector, the diffraction exhibits a high degree of symmetry between the positive and negative first orders. We present the diffracted intensity as a function of tilting angle and radial position on the lens. A similar data presentation limited to one order of diffraction was reported previously $[7,8]$. ImageJ [17] was found to be very useful to view the data. We present the data in Fig.2 in a so-called stacked orthogonal view, in which the diffraction at different rocking angles are stacked vertically. This provides an efficient visualization of the uniformity of diffraction angles as function of d-spacings which, in turn, are a function of deposition thickness. This way of representing the data enables initial screening for defective regions [13]. 


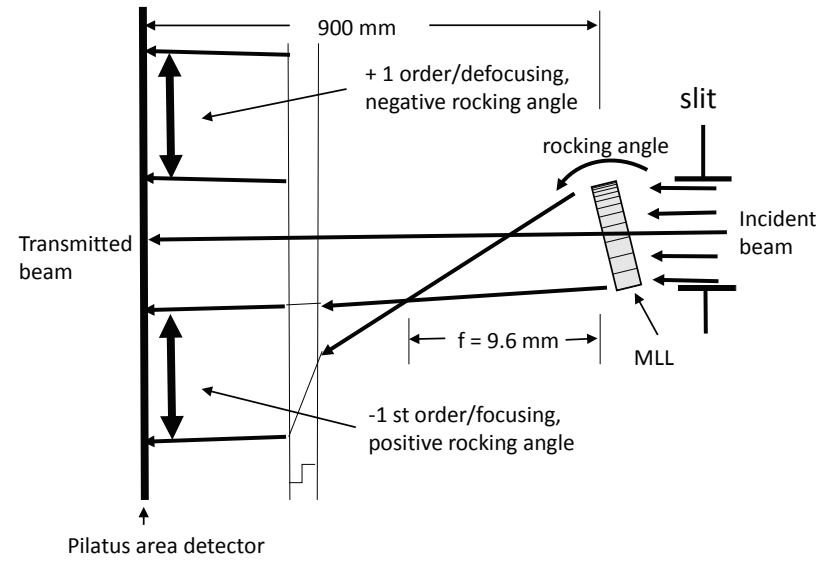

FIG. 1. Experimental arrangement for diffraction measurements. The schematic view is from above. The focusing order is shown. The defocusing order has a virtual focus 9.6 $\mathrm{mm}$ upstream of the lens. However, since the distance to the area detector of $900 \mathrm{~mm}$ is much larger, the far-field patterns of both orders are effectively symmetric. A scintillator plus CCD (not shown) was placed $330 \mathrm{~mm}$ downstream of the MLL to image the transmitted beam. By means of images on the CCD, a slit was set to illuminate only the full MLL. That is, the MLL was neither underfilled nor overfilled.

The sloping behavior of the diffraction peak with rocking angle arises because by design there is a monotonic decrease in zone width, and, consequently, increase in Bragg angle, as one proceeds from inner zones to outer zones. We will refer to this as the position on the lens. For an ideal MLL of the present design, i.e., with all parallel interfaces, the orthogonal view in Fig. 2 would show a linear dependence of the Bragg angle on position, as indicated by the dashed line in Fig.2 [13]. This also follows directly from the zone plate law [18]. However, as shown in Fig.2 the peak diffraction as a function of position deviates from the dashed line, and this indicates that there is bending [13]. We note that wedged MLLs which have interfaces that are angled to preserve a Bragg condition would ideally have a purely horizontal diffraction pattern for the focusing order in a plot such as Fig.2 [19]. Wedging is desired for increased efficiency since an increasing number of zones come into the Bragg condition at an optimum rocking angle. Bending strain can result in effective wedging [20], and such wedging would result in a distinct asymmetry between the negative and positive first orders [19]. We infer that in the present case

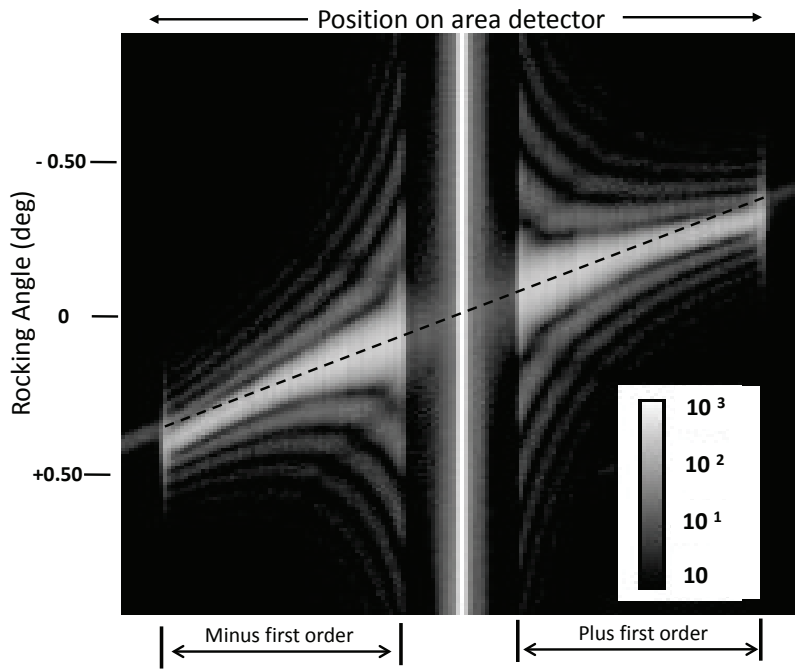

FIG. 2. Diffraction recorded for negative and positive first orders. The MLL was set to diffract horizontally. The abscissa corresponds to horizontal distance along the detector, and the ordinate is for the rocking angle. The vertical stripe in the center is the transmitted beam. We defined the zero of angle as the crossing point between the dashed line and the vertical line for the transmitted beam. The thicker inner zones contribute to the diffraction nearer to the transmitted beam for both orders, and diffraction from the thinner outer zones occurs at larger absolute rocking angles. An artificial diagonal dashed line is included to show that there is some bending of the lens since the diffraction should follow the straight dashed line in the absence of bending.

a slight bending was present, but the attendant wedging is not pronounced.

We also observed an extensive fringe system for both orders. These fringes were used to determine the section thickness. We determined a section thickness of $9.6 \mu \mathrm{m}$ using a similar method of analyses as that reported earlier for Laue-case diffraction from a sectioned multilayer [21].

Measurements with the lens withdrawn were also made to allow calculation of the measured efficiency. As is well known for MLLs, the efficiency is a strong function of rocking angle and exceeds that of a conventional Fresnel zone plate because dynamical diffraction (also called volume diffraction) is applicable for zones having a small deposition thickness. Consequently, satisfying the Bragg condition yields high efficiency. The efficiency was obtained by summing over all pixels corresponding to the aperture of the lens for each rocking angle and normalizing to the total counts obtained with the MLL withdrawn. A slit was set to aperture only the full MLL. This allowed a calculation of the efficiency. The dependence of the efficiency on rocking angle is show in Fig. 3. 


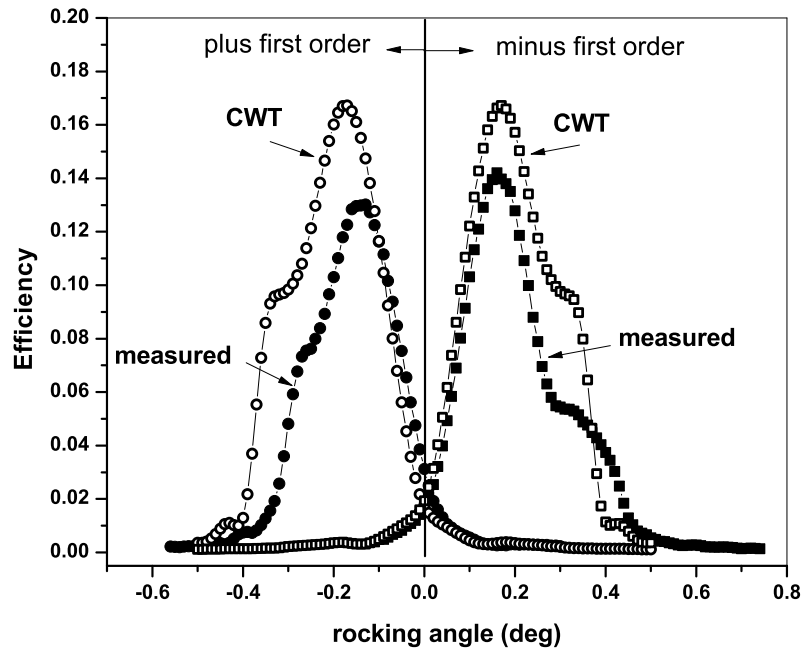

FIG. 3. Effiency as a function of rocking angle. The results of coupled wave theory are also shown.

Also shown in Fig. 3 are the results of coupled wave theory (CWT) simulations for an ideal lens in accordance with its design [22]. For the calculations optical parameters based on bulk material densities were applied [23].

As reasonably expected, the measured efficiencies are somewhat smaller than predicted by CWT. CWT predicts perfect mirror symmetry for the plus and minus orders as seen in Fig.3. The asymmetry between the orders is an indication of bending as discussed above. An intriguing and unexpected aspect of both the data and the CWT simulation is the occurrence of shoulders in both orders. These shoulders arise for diffraction from the thinner zones and can be explained as a manifestation of a transition from kinematical to dynamical diffraction between inner and outer zones. Rocking curves narrow as the dyamical diffraction regime is approached. In the case of dynamical diffraction, rocking curves are narrower. This results in a lower efficiency as the MLL is rocked to diffract from the outer zones.

Summarizing, we report on the efficiency of an MLL comprised of $\mathrm{WSi}_{2} / \mathrm{Al}$ layers stacked to a multilayer stack thickness of 102 microns, which roughly doubles the previous record aperture for an MLL. There were no defects in the layer structure for the portion of the MLL reported on here, but a slight overall bending was observed. The measured efficiencies compare well to results of coupled wave theory. Measurement of the focus are planned.

We acknowledge Dr. N. Kujala for assistance in the early phases of this work, and we thank H. Yan for commenting on the manuscript. We are grateful to the X-ray Science Division of the Advanced Photon Source for support. This work was supported by the U.S. Dept. of Energy, Office of Basic Energy Sciences, under Contract No.
DE-AC-02-06CH11357. Work carried out at National Synchrotron Light Source II and the Center for Functional Nanomaterials at Brookhaven was supported by the US Dept. of Energy, Office Basic Energy Sciences under contract DE-SC00112704. Work at Fraunhofer IWS was partly funded by the European Union (ERDF) and the Free State of Saxony via the ESF project 100087859 (ENano).

${ }^{a}$ Corresponding author: atm@anl.gov

[1] H.C. Kang, J. Maser, G.B. Stephenson, C. Liu, R. Conley, A.T. Macrander, and S. Vogt, Phys. Rev. Lett. 96, 127401 (2006).

[2] H.C. Kang, H. Yan, R. P. Winarski, M.V. Holt, J. Maser, C. Liu, R. Conley, S. Vogt, A.T. Macrander, and G.B. Stephenson, Appl. Phys. Lett. 92, 221114 (2008).

[3] H.C. Kang, G. B. Stephenson, C. Liu, R. Conley, R. Khachatryan, M. Wieczorek, A. T. Macrander, H. Yan, J. Maser, J. Hiller, and R. Koritala, Rev. Sci. Instrum. 78, 046103 (2007).

[4] R. Conley, C. Liu, C.M. Kewish, A.T. Macrander, and C. Morawe, Proc. SPIE 6705, 670505 (2007); R. Conley, C. Liu, . Qian, C.M. Kewish, A.T. Macrander, H. Yan, H.C. Kang, J. Maser, and G.B. Stephenson, Rev. Sci. Instrum. 79, 053104 (2008).

[5] H. Yan, V. Rose, D. Shu, E. Lima, H. Kang, R. Conley, C. Liu, N.Jahedi, A. Macrander, G. Stephenson, M. Holt, Y. Chu, M. Lu, and J. Maser, Optics Express 19, 15069 (2011).

[6] H. Yan, R. Conley, N. Bouet, and Y. Chu, J. Phys. D: Appl. Phys. 47, 263001 (2014).

[7] T. Koyama, S. Ichimaru, T. Tsuji, H. Takano, Y. Kagoshima, T. Ohchi, and H. Takenaka, Applied Physics Express 1, 117003 (2008).

[8] T. Koyama, H. Takenaka, S. Ichimaru, T. Ohchi, T. Tsuji, H. Takano, and Y. Kagishima, AIP Conf. Proc. 1365, 24 (2011).

[9] Y.S. Chu, C. Liu, D.C. Mancini, F.De Carlo, A.T. Macrander, B. Lai, and D. Shu, Rev. Sci. Instrum. 73, 1485 (2002).

[10] J. Maser, G.B. Stephenson, S. Vogt, W. Yun, A. Macrander, H.C Kang, C. Liu, R. Conley, Proc. SPIE 5539, 185 (2004).

[11] X. Huang, H. Yan, E. Nazaretski, R. Conley, N. Bouet, J. Zhou, K. Lauer, L. Li, D. Eom, D. Legnini, R. Harder, I. Robinson, and Y. Chu, Sci. Rep. 3, 3562 (2013).

[12] A. Kubec, S. Braun, S. Niesse, P. Kruger, J. Patommel, M. Hecker, A. Leson, and C.G. Schroer, J. Synchr. Rad. 21, 1122 (2014).

[13] Adam Kubec, Naresh Kujala, Raymond Conley, Timothy Mooney, Deming Shu, Jeffrey Kirchman, Kurt Goetze, Nathalie Bouet, Juan Zhou, Jorg Maser and Albert Macrander, in preparation.

[14] A. Macrander, M. Erdmann, N. Kujala, S. Stoupin, S. Marathe, X. Shi, M. Wojcik, D. Nocher, R. Conley, J. Maser, and L. Assoufid, in preparation.

[15] D. Shu, H. Yan, and J. Maser, "Multidimensional alignmnet apparatus for hard x-ray focusing with two multilayer Laue lenses" , US patent No. 7,597,475 (2009). 
[16] P. Kraft, A. Bergamaschi, Ch. Broennimann, R. Dinapoli, E.F. Eikenberry, B. Henrich, I. Johnson, A. Mozzanica, C.M. Schleputz, P.R. Willmott, and B. Schmitt, J. Synchr. Rad. 16, 368 (2009).

[17] Caroline A. Schneider, Wayne S. Rasband, and Kevin W. Eliceiri, Nature Methods 9, 671 (2012); http://imagej.nih.gov/ij/ .

[18] N. Jahedi, R. Conley, B. Shi, J. Qian, K. Lauer, and A. Macrander, Nucl. Instrum. Meth. A 616, 89 (2010).

[19] X. Huang, R. Conley, N. Bouet, J. Zhou, A. Macrander, J. Maser, H. Yan, E. Nazaretski, K. Lauer, R. Harder, I.K. Robinson, S. Kalbfleisch, and Y.S. Chu, Optics Ex- press 23, 12496 (2015).

[20] Sven Niese, Peter Krüger, Adam Kubec, Roman Laas, Peter Gawitza, Kathleen Melzer, Stefan Braun, and Ehrenfried Zschech, Thin Solid Films, 571, 321 (2014).

[21] H.C. Kang, G.B. Stephenson, C. Liu, R. Conley, A.T. Macrander, J. Maser, S. Bajt, and H.N. Chapman, Appl. Phys. Lett. 86, 151109 (2005).

[22] J. Maser and G. Schmahl, Optics Communications 89, 355 (1992).

[23] Burton L. Henke, Eric M. Gullikson, and John C. Davis, Atomic Data and Nuclear Data Tables 54, 181 (1993). 